\title{
Consequences of Choice : Exploring Successful and Unsuccessful Decision-Making Attributes in UK Homicide Investigations
}

\section{Howard Neil Atkin}

\begin{abstract}
Flawed decision-making is widely identified as having led to miscarriages of justice and failed homicide investigations. This research explores decision-making by Senior Investigating Officers in UK homicide investigations, including where initial investigations were unsuccessful, or resulted in wrongful convictions. It offers a new 'investigative continuum' perspective as a means to explore decisions and for linking the impact of decisions to successful or unsuccessful outcomes. Using this model, it correlates 'explorative', 'creative' and 'impactive' decisions with positive investigative outcomes. It corroborates 'tipping points' in decision-making processes, identifies a new feature, 'Transition Points', and observes how these can affect investigative outcomes.
\end{abstract}

Key words: Homicide Investigation; Homicide Review; Decision-Making; Cold Case; Senior Investigating Officer; Tipping Point; Transition Point; Investigative Continuum

\section{Acknowledgements}

Thanks are due to Professor Jason Roach, Dr Michelle Rogerson (University of Huddersfield), and Dr Rachael Aplin (University of Newcastle) for useful discussions in preparing this article.

\section{Introduction}

Flawed decision-making has repeatedly been identified as responsible for failed homicide investigations and miscarriages of justice, (Byford Report,1981; Stephen Lawrence Inquiry Report,1999; Shipman Inquiry Report, 2005). Multiple studies have found the most common error in crime investigation to be decision-making (Nicol, Innes, Gee \& Feist, 2004). 
A decision can be defined as '-a choice between a variety of alternatives', involving decisionmakers in a, 'process of identification, analysis, assessment, choice and planning', (Heller, 1998, p.26). Extant research into factors affecting decision-making broadly focusses on two areas; 'process', and 'context'.

Regarding 'process', logic and critical thinking are highlighted as methodological processes that support 'effective decision-making', (Copi \& Cohen, 1998, p.3; Fisher, 2001). Competing hypotheses are highlighted as a mechanism to refine mental models and analyse complex problems, (Heuer, 1999), and 'scientific method', logic and reasoning to facilitate analysis of investigative data, (Atkin, 2000; 2013). In summary, effective decisions result from systematic processes, with clearly defined elements in sequential steps.

Regarding 'context', studies highlight how different physical and mental states can affect perceptions -'models' - of physical reality created by the human mind, (Popper (1972), and how factors like cognitive bias and 'framing' can affect decision-making, (Khaneman, 2011; Sinclair, 1984). Research into the impact of bias and subjectivity upon investigators decisionmaking highlights factors including time pressure, and context, (Rossmo, 2006; Dror, Busemayer \& Basola, 1999), a view developed by Kahneman and Tversky (2000) who present the concept of 'framing' - considering information from a singular perspective rather than other potentially more useful perspectives. Studies have also considered how decision-making impacts upon homicide investigative outcomes, noting how in operational settings individuals utilise their experience to make decisions (Stelfox \& Pease, 2005; Ask \& Granhag, 2007; Rossmo, 2008). In summary, 'context' can introduce subjective variability and bias into decision-making. Moreover, this potentially conflicts with the structural 'process' concept identified earlier. 
Homicide investigation might usefully be considered as essentially a cyclical 'problem-solving' process, that collects information in a focussed way and makes decisions based upon that information that support further information collection.. The 'process objective' is to achieve particular goals, ideally the successful conclusion of an investigation, or as a minimum the exploration of all reasonable lines of enquiry. UK Government guidance structures such processes (MOD, 2011), whilst UK Police Forces traditionally utilise generic problem-solving models, notably 'SARA' (Weisburd et.al., 2008), and the 'National Decision-Making Model' (ACPO, 2012), as good practice. All emphasise the importance of decision-making.

Effective decision-making is also reflected throughout current UK Police Core Investigative Doctrine, which highlights a core skill for any investigator as, '...the ability to make decisions which can be justified to others', identifies, decision-making as 'central to any criminal investigation' (ACPO, 2005, p.58), and promotes the concept of an 'investigative mind-set' (ACPO, 2005, p.60).

This doctrinal guidance highlights decision-making as a key component of homicide investigation, as a key investigative driver influencing the outcome of an investigation, and as an important attribute for investigators and investigative managers, in particular the Senior Investigating Officer (SIO). Significantly, it acknowledges that decisions and actions taken in an initial investigation must inevitably impact upon any subsequent re-investigation, alludes to the fact that a single 'homicide' may in fact be subject to several investigations, for example when initial investigations are unsuccessful, or result in 'wrongful' convictions, noting that the first opportunity to examine a source of material and test its reliability may be the last, and acknowledging that to pass up such opportunities may mean that they are 'lost forever', (ACPO, 2005, p.63).

Some authors (e.g. Roycroft, 2009) note how the decision-making 'mind-set' of homicide investigators can be influenced by critical factors in the investigative process. Also, authors 
(Atkin \& Roach, 2015; Roach, 2017) highlight that different psychological and contextual factors exist which can facilitate bias in investigator decision-making in cold case (undetected) homicides, -including 'the biasing effect of inheriting a chain of decisions, ...- made by, ...previous investigators, and the negative framing effect that the term 'cold case' can have on investigator confidence and on their subsequent decision-making' (Roach, 2017, p.1).

Another relevant perspective is that of decision-making being influenced by 'group' or 'collaborative' factors. Sennett (2012) identifies murder investigations as complex social systems, 'replete with little dramas of association and deference' (Sennett, 2012, p.136). Innes (2002) observes how standardised activity sequences of homicide investigators can be viewed as a social process where factors including law, incident circumstances and police 'organisational properties' can shape investigator actions and combine to influence investigative processes. Innes \& Brookman (2013) suggest that 'social systems', comprising distinctive components working together to accomplish a larger task, applies to homicide investigators.

Authors also note how the impact of the SIO and their decision-making can have a significant impact upon both the investigative process and upon investigative outcomes, (Stelfox \& Pease, 2005; Rossmo, 2008). Stelfox (2006) notes that '...the investigation process does not consist of a series of designed activities... -but is a loose framework within which officers deploy a range of investigative techniques, knowledge and decision making skills depending on the needs of the individual case.' (Stelfox, 2006, p. iii). Studying SIO decision-making in actual homicide investigations, Roycroft (2009) highlights investigative strategies focussed upon thematic 'solving factors' as supporting successful investigative outcomes. If correct, this infers that factors other than those that might be described as objective, or derived from good practice or process, might influence investigations and outcomes, and further, that outcomes might reflect subjective, individual factors including personal and confirmation bias by investigators, and perhaps most importantly, the SIO. 
Studying decision-making sequences in murder investigations, Innes and Brookman (Innes 2003; Innes \& Brookman 2013) note that early investigative decisions significantly frame the conditions of possibility for subsequent decisions, noting each decision point within an investigative process can open up or close down certain lines of action. Exploring the 'intuition' of homicide detectives, Wright (2013) identifies 'cycles of cognition' within thought processes of subjects presented with a range of typical 'successful' investigations, thus supporting the 'concatenated decision model' observed by Innes and Brookman. In their studies of sequential decision-making by SIO's, Fahsing \& Aske (2013) further develop this concept of decision-making as a sequential process, identifying 'tipping points' - points in time when the mind-set of the SIO 'switched' from 'exploratory' (seeking to identify a suspect or suspects) to 'verification' (seeking to verify a suspect's involvement in the crime).

Thus in summary, extant research and doctrine highlight features of personal and organisational factors influencing decision-making, in general and within homicide investigations, as follows:

1) They are complex and highly subjective processes, within which different mechanisms may exist for sequential decision-making, mental frameworks and 'thinking-processes'; these may change over time, both during and between different investigative phases, for example at 'Tipping Points' when investigations first identify specific suspects, and these factors can impact upon investigative outcomes.

3) Beyond the individual decision-makers, organisational processes involved are also complex, often formulaic and even institutionalised. Different methodologies may exist for routine or 'initial' investigations, -the 'self-solvers', and subsequent investigations of undetected or wrongly detected homicides - the 'whodunits'

4) Processes and frameworks utilised may themselves may also influence investigative decision-making and outcomes, both at individual and organisational levels. 
Significantly, much of the 'focus' and the methodology of earlier research has been based upon exploring 'decision-making' as a process conducted by an individual decision-maker as against it being a group or an 'organisational or process-driven' activity. Further, where studies tested decision-making relating to homicide investigation, many based their analysis upon responses to entirely artificial contrived case examples. As such, might be described as being relatively simplistic compared to real cases. As such, they may arguably miss the potential to examine the holistic environment in which decision-making takes place, and also in ecological validity. As such, extant research, both in terms of its outcomes and its restrictions, suggests a number of areas worthy of further study.

First, the question arises as to what factors affect an individual's decision-making, and how. Second, linking the concepts of decision-making as a sequential process, moreover one that exhibits 'tipping points' at different stages in the sequence, raises the possibility these two factors may be linked. Third, if these factors and processes do affect individual-decisionmaking, they may also affect group or organisational decision-making, such that individual and group/organisational decision-making might influence each other. Fourth, given the oftensignificant differences in the length of investigation for initial investigations relative to historic cases, any understanding of historic investigation might be influenced by factors such as perspective and context, difficult to identify and assess, and to a greater extent than initial investigations. And finally, how do any of these processes impact upon decision-making in the real world of homicide investigation rather than in the restricted confines of 'laboratory exercises', generally using artificial cases. 


\section{Methodology}

Extant research into subjective processes in homicide investigations having focussed upon analysing responses to hypothetical scenarios, one objective of this research was to explore the form, flow and impact of decision-making in real UK homicide investigations, successful and unsuccessful, in order to better understand factors that may influence investigative outcomes, and how decision-making in initial investigations might influence the outcomes of subsequent re-investigations. Decision-making in homicide investigations ultimately depending upon human choices suggested a phenomenological research approach and qualitative methodology, seeking to identify the qualities of a phenomenon (Langdridge \& Hagger-Johnson, 2013), and from the perspective of the subjects under study (Corbin \& Strauss, 2015).

\section{Conceptual Frameworks: applying an 'Investigative Continuum’ Perspective}

Conventional practice within law enforcement categorises cases as current or historic, 'open' or 'cold', depending primarily on their position within an organisational timeline. However, for each individual case, such artificial and arbitrary categorization or classification may be unhelpful or even harmful to the potential 'success' of that investigation, in terms both of progress or outcome; this represents a potentially significant conceptual failing within current law enforcement practice.

Consider an individual homicide case. The investigative process occurs along a single timeline. The start point of that investigative timeline is the point when a homicide is first allocated for investigation, (ACPO 2006). That will always be at a point in time after the 'homicide event' occurred, and the investigation will routinely explore a period of time even before and leading up to the 'homicide event' in an effort to discover relevant precursor information. Once the investigation commences, it will proceed in time until a point where it either is 'closed', (generally because it is 'successful'), or where otherwise it enters a 'cold case' process for 'cold case'; review and/or re-investigation. Again this will routinely continue 
until it is successfully resolved and thus becomes 'closed'. Throughout this investigative timeline, the investigation (or re-investigation) will also routinely be subject to a formal review process, (e.g. ACPO 1998; 2010). Even when 'closed', investigations are routinely maintained beyond that point in time to allow for the possibility of legal appeal against an outcome. Finally, the possibility exists that at some future point in time some new 'event' may result in a 'closed' investigation being revisited and 'reopened' -reviewed or re-investigated, for example where a wrongful detection or conviction in that case comes to light, thus extending the timeline of that case.

A more representative way of viewing a single case might thus be that it is subject over time to what might be termed an 'investigative continuum', -a process of investigation, review, and possibly reinvestigation, -which rather than a set of discrete events is actually one continuous process, in time at least. This certainly could be argued as the viewpoint of many of the key stakeholders other than Law Enforcement; for victims, victim families, witnesses, the public at large, even suspects and other agencies, from the moment it occurs a homicide is arguably a single on-going experience in their lives and remains so largely regardless of how it is categorized by Law Enforcement. This conceptual perspective, differing as it does from organisational Law Enforcement, is important in that it invites different approaches both to data and research, than those routinely conceived.

Utilising an 'investigative continuum' contextual framework provides new opportunities to view a single case as relevant to different categories at different points in time along the continuum. This approach also offers the potential for data from a single case to be more representative of these categories, and new potential to explore the continuity of a single case as it moves between categories, and thus to explore factors and rationales behind these transitions and the impact of these transitions upon the investigation, and thus to identify causation and effect, be that beneficial or detrimental to eventual outcomes, in a far more direct and effective way than might be the case if these changes were merely explored by comparing events in different 
cases. This approach may thus help focus analysis towards identifying which, if any, elements of investigation or review were the cause for individual cases to move from being 'unsuccessful' to 'successful', or vice versa, the ultimate goal of this research.

\section{Case Sampling; using a 'Selection Matrix'}

Initial research design anticipated detailed study of a representative sample of cases of homicide investigation, including current and historic cases. The research objective was to try and identify cases where appropriate and comparative analysis might offer new insight into how, and why, investigative approaches and processes might have differed, and might have affected what might be termed 'successful' outcomes. Full access was obtained to all electronic and paper-based investigative records pertaining to 43 ongoing Homicide investigations from a major urban UK Police Force, including current, 'initial' investigations and 'cold cases' subject to review and re-investigation. This dataset was mainly documentary in nature and textual in structure. The sheer volume of data made detailed analysis of every one of the $(n=43)$ cases impractical, so selection of a representative sample of cases was necessary. That being the case, the first research issue became one of sample selection. Applying the 'Investigative Continuum' conceptual framework usefully identified three separate case types, in part based on their status at a particular point in time, namely the commencement of the research, and highlighting the need to consider three case types, namely :

- 'successful' cases;

- 'unsuccessful cases, and;

- 'wrongful investigations', -cases that whilst initially categorised as 'successful', through further investigation were found to have been wrongfully categorised.

Within social science research, Langdridge \& Hagger-Johnson (2013, pp.54-57) observe, that in qualitative research, non-random sample selection is often deliberately used either for 
practicality, or to select cases that might illustrate a putative theory, or both. They also identify 'opportunity' or 'convenience' sampling as particularly useful, defining these as sampling 'in any way possible (or convenient)', (2013, p.56). Bryman (2016) highlights purposive sampling in qualitative research, describing it as a non-random technique, who's goal is to sample '...in a strategic way, so that (samples) are relevant to the research questions... -posed', (p.408409). Building upon this approach, Glaser \& Strauss highlight theoretical sampling as, '...the process of data collection for generating theory whereby the analyst jointly collects, codes, and analyses his data and decides what data to collect next and where to find them in order to develop his theory as it emerges. The process of data collection is controlled by the emerging theory, whether substantive or formal', (1967, p.45).

The research questions suggested a generic purposeful approach to case sampling, moreover, one that also supported a theoretical sampling methodology whereby each stage supported testing of emerging theoretical ideas. However, whilst these were the prime drivers for sampling, other elements also had influence. For example, focussing upon cases from a single police force introduced an element of opportunistic convenience sampling. Similarly, within purposeful sampling types, the focus of the research questions towards developing theory based on specific dimensions of interest, notably differences in methodology and outcomes and their causation, emphasised elements in particular of typical, critical and even stratified purposive case sampling, whilst practicality in terms of data volume tended to eliminate criterion sampling.

Alongside the reality of trying to predict how each case might represent the type of homicide investigation of interest to the research, a second 'practical' issue relevant to case selection was the quality and range of data available for analysis within each case. To address this, a 'selection matrix' was constructed to enable those features to be compared. This assessed each case based on its potential value to the research questions and based on the value of key data content in terms of the case following an 'investigative continuum'. These features 
were 'scored' based on their possession of features in two key groupings, case data 'content' -the relative presence of substantive data in key variables, and case data 'significance' -the relevance of the case to the overarching research objective to evaluate cases from the three key 'categories'; using this method, the best six cases (two from each category) were selected for detailed study.

\section{Decision Data : Codeification and Categorisation}

Initial scoping showed the case data 'form' to be primarily textual, recording observed caserelated facts or information, or human interpretations of that information, primarily investigative 'value judgements', namely decisions made directing the conduct of the investigation, or about how the investigation was being or had been conducted, for example Review observations and recommendations. Whilst both information and value judgements understandably differed between cases, the research objective was to identify patterns and issues within the dataset factors that may influence (or not) the outcome of the process, be that positive or negative. As such, this could be categorised as thematic analysis (King, 1998).

Whichever the model adopted, the decision-making process essentially consists of two key components; information and decisions. This was reflected in the case data, with decisions in the form of SIO Policy logs or Review recommendations and information associated with those decisions present in a range of forms. That being so, some form of codification, categorisation- was useful to the research to systemise individual case data into a form suitable for thematic analysis.

This categorisation grouped all case data into two overarching categories, namely material that was either 'decision' or 'information'. Since the research aim was to seek to identify factors that influenced the outcome of investigations, a logical approach was to sub-categorise 'decision' data based upon the type and impact of the decision, and to subcategorise 
'information' on the basis of how it influenced particular decisions. Accordingly a subcategorisation matrix was constructed and applied (See Table 1).

Initial scoping also identified that some singular 'policy decisions' consisted of several different decisions, often in more than one of the above categories. Clearly the individual features of each decision in a singular 'clustered decision' might mask the importance of those features. Accordingly, where a policy decision contained multiple decisions, these were separated out as 'sub decisions' within the overall policy decision and categorised individually.

The available material for each of the 6 selected cases that evidenced decision-making was then codeified. The resulting data matrix captured and codeified a total of over 4000 individual decisions into 6 decision categories and 13 sub-categories. This feature enabled limited quantitative exploration of the summary data. For example, totalling the number of decisions made in each case by category and subcategory enabled quantitative comparisons between decisions made, and the three different case types. To better attempt to observe differences between case types, the total decisions in each category for both cases per case type were combined and an average calculated to derive an arithmetic mean. However, whilst this supported comparative analysis, both the absolute total number and any derivation from that total of any category or case type would differ simply based on the total number of decisions made, and thus potentially preclude meaningful comparisons. In order to attempt better comparisons, the arithmetic mean values numbers and derivatives for each category and case type were divided by the total number of decisions for each category and then multiplied by 100 to arrive at a percentage value, thus enabling each to be be compared as a relative proportion of the total number of decisions by case type. 


\section{Results and Discussion}

Even this simple overview, (Chart $A)$ offered several interesting observations. The mean proportions of decisions per case type divided by the number of categories showed marked differences in that 'Potentially Successful' re-investigations' $(19.8 \%: n=257.5)$ featured almost $50 \%$ more decisions overall compared to 'Unsuccessful' re-investigations' (28.4\% : n=369.6). If decisions are related to 'success', that may infer that, in general, greater decision numbers correlate directly with greater success. However, 'Wrongful Initial Investigations' $52.8 \%$ : $n=687.1$ ) featured almost $50 \%$ more decisions than 'Potentially Successful' re-investigations $28.4 \%: n=369.6$ ). Clearly that might appear to conflict with the previous inference in that despite these being wrongful initial investigations, greater decision numbers apparently did not result in greater success.

However, in such cases, even though the initial investigations were 'wrongful' and thus flawed, the eventual re-investigative outcomes were 'successful'. Further, in an 'initially wrongful' investigation it may be likely that higher numbers of decisions would be made, if only as a reflection of the added attention and care such investigations attract given they are exploring not just an undetected offence, but what is also potential organisational failure of a magnitude wholly different and higher than for an unsuccessfully investigated case. Thus stakeholders may be more likely to expect and deliver investigations with more decisions in wrongful cases rather than cases which have always merely been 'undetected' simply because of the greater risk to reputation and to society in general and thus a desire to document decisions taken in far more detail, rather than to actually make far more decisions than in other investigation types.

Beyond these generic comparisons, examining differences across case types for each individual decision category offered further insight, as follows: 
Decision 'Objectives' : 'Objective' decisions were sub-divided into one or more of three objectives (Descriptive ; Administrative ; Investigative). This categorisation recognised that a single decision might have multiple objectives, for example an 'investigative' decision might also identify consequential 'administrative' and/or 'investigative' decisions. Such decisions might thus score in one or more sub-categories, so that such multiple scores indicated more than one objective for a single decision and thus arguably a higher level of complexity. An advantage of this categorisation process was that each individual sub-category could be compared both relatively against other sub-categories, and as a proportion, -a percentage,of the total decisions for that sub-category.

This identified a number of differences, both between the type (sub-category) of objective (Descriptive : Administrative : Investigative) and also between the type of case. In general terms, investigations generated higher, broadly similar proportions of decisions with 'descriptive' and 'investigative' objectives than with 'administrative' objectives, a pattern that occurred regardless of case type. However, comparing 'Unsuccessful' and 'Potentially Successful' case types, whilst all three categories of decision objective were higher for 'Potentially Successful' re-investigations (cumulative values 58\%:42\%:n=116.8: $n=-84.5$ ), the relative proportion of 'investigative' decisions was much higher in 'Potentially Successful' investigations than for 'Unsuccessful' investigations (cumulative values $63 \%: 37 \%: n=52.8$ : $n=31$ ). Further, when comparing 'wrongful investigations' with other case types, all three categories of decision objective for 'wrongful investigations' were much higher than for other case types (cumulative values $67 \%: 33 \%: n=237.1: n=1116.8$ ). This might be explained by a desire to document decisions taken in more detail than might be the case for other investigation types, rather than to actually make far more decisions than in other investigation types, as identified earlier, -a form of administrative 'tick boxing'.

Decision 'Causes' : Decision 'Causes' were sub-categorised into 'Proactive', -generated by some creative thought or initiative from within the Investigation, or 'Reactive', -generated as a 
reaction to some externally driven event or information. The proportion of 'Proactive' decisions was markedly higher for 'Potentially Successful' re-investigations than for 'Unsuccessful' reinvestigations, $(n=6.5: n=20.4 ; 24 \%: 76 \%)$, and markedly higher for 'Wrongful Initial' investigations than for 'Potentially Successful' reinvestigations $(n=20.4: n=37.9 ; 35 \%: 65 \%)$. This may infer that higher proportions of 'proactive' decisions correlate with greater investigative 'success'. Regarding 'Reactive' decisions, the proportion was slightly lower for 'Potentially Successful' reinvestigations than for 'Unsuccessful reinvestigations ( $n=39.5$ : $n=39.2$ ). If that occurred across all case types, that might infer that as proportions of 'Proactive' decisions' increased, 'Reactive' decision proportions fell. However, the proportions for reactive decisions for 'Wrongful Initial' investigations were markedly higher, almost double that for either of the other case types $(n=39.5, n=35.2: n=61.2)$. This may infer a conflict between two principles, in that whilst there is a trend away from 'Reactive' towards 'Proactive' decisions in the case of 'Potentially Successful' re-investigations, in the case of 'Wrongful Initial' investigations this trend is simply overridden by the generic increase in proportions of all decisions already observed in such cases.

Decision 'Intent' : This categorisation sought to distinguish between decisions that were 'exploratory', -acting to expand the investigation from that point forwards, versus those that were 'regressive', -acting to limit the investigation going forwards. 'Expansive/explorative' decisions were present in higher proportion in 'Potentially Successful' re-investigations compared to 'Unsuccessful' reinvestigations ( $n=12.5: n=28.4,=31 \%: 69 \%)$, and similarly in 'Wrongful Initial' investigations compared to 'Potentially Successful' reinvestigations ( $n=28.4$ : $\mathrm{n}=53.4,=35 \%: 65 \%$ ). By contrast, 'regressive' decisions were present in slightly lower proportions when comparing 'Potentially Successful' re-investigations to 'Unsuccessful' reinvestigations and similarly when comparing 'Wrongful Initial' investigations to 'Potentially Successful' re-investigations. This may infer a direct correlation between the relative proportion of 'expansive/explorative' decisions and greater investigative 'success'. 
Decision 'Connectivity' : This categorisation sought to distinguish between different decisions on the basis of their being independent of each other, or alternatively having a relationship where one decision of itself led directly to others rather than the arising from fresh information. Whilst the proportion of 'related' (consequential) 'and 'unrelated' (linked) decisions was present in higher proportion in 'Potentially Successful' reinvestigations compared to 'Unsuccessful' reinvestigations and also in 'Wrongful Initial' investigations compared to 'Potentially Successful' reinvestigations, there were no apparent anomalies in this relationships between case type, which whilst this may infer a direct correlation between the relative proportion of both 'connected' and 'unconnected' decisions and greater investigative 'success', actually may in fact merely be a direct reflection of the earlier correlation between greater decision numbers and 'success'; in other words, this categorisation of itself was not apparently a factor correlating with 'success'.

Decision 'Creativity' : This categorisation sought to identify where the stimulus for a decision was 'creative thought' rather than just a response to information; in effect the application of Inductive as against Deductive Logic to think 'outside the box'. A much higher proportion of 'creative' decisions' were found in 'Potentially Successful' re-investigations compared to 'Unsuccessful' reinvestigations, $(n=7: 18.4,=28 \%: 72 \%)$ and also in 'Wrongful Initial' investigations compared to 'Potentially Successful' reinvestigations, $(n=18.4: 39.7,=32 \%$ : $68 \%)$, This may infer a direct correlation between the relative proportion of 'creative' decisions and greater investigative 'success'.

Decision 'Impact' : This categorisation sought to explore two elements of the same categorisation, namely decisions that having some clear impact on the investigation. A much higher proportion of 'impactive' decisions were seen in 'Potentially Successful' reinvestigations compared to 'Unsuccessful' reinvestigations, $(n=31: n=47.6 ; 39 \%: 61 \%)$ and also in 'Wrongful Initial' investigations compared to 'Potentially Successful' reinvestigations, $(n=47.6$ $: n=87.9 ; 35 \%: 65 \%)$. This may infer a direct correlation between the relative proportion of 
'impactive' decisions and greater investigative 'success'. Where 'impactive' decisions were identified, a secondary question arose of what proportion could be categorised as having a 'positive' or 'negative' impact on the investigation. In the case of decisions with a 'positive impact', a much higher proportion of 'positive impact' decisions were seen in 'Potentially Successful' reinvestigations compared to 'Unsuccessful' reinvestigations, $(n=8.5: n=20.4$; $29 \%: 71 \%$ ) and also in 'Wrongful Initial' investigations compared to 'Potentially Successful' reinvestigations, $(n=20.4: n=59.5 \% ; 25 \%: 75 \%)$, whilst in the case of decisions with a 'negative impact', a much lower proportion of 'positive impact' decisions were seen in 'Potentially Successful' reinvestigations compared to 'Unsuccessful' reinvestigations, $(n=17$ : $n=10.8 ; 65 \%: 35 \%)$ and also in 'Wrongful Initial' investigations compared to 'Potentially Successful' r-investigations ( $n=10.8: n=8.6 ; 56 \%: 44 \%)$. This may infer that where decisions are impactive, the proportion having a 'positive impact' on an investigation correlates directly with increased 'success' whilst the proportion having a 'negative impact' has an inverse correlation with 'success'.

\section{Exploring the 'Investigative Continuum'}

The 'Investigative Continuum' perspective offered opportunities to explore how the proportions of different decision categories varied over the lifetime of the 'investigation', and to explore relationships between contemporaneous decisions and contemporaneous outcomes. The data coding matrix created for the case studies coded decisions contemporaneously, so the visual presentation of the data matrix (using MS Excel) enabled the coded variables for each decision to be visualises, such that the coding matrix itself offered useful opportunities to visualise changes over time and to identify time-related trends, features and patterns of interest, as follows:

Decision 'Objectives' over Time : Examination of the patterns in all three sub-categories of decision 'objectivity' showed broadly the same pattern regardless of case type, namely that all sub-categories were distributed evenly over the whole period of the investigative process, 
other than 'Unsuccessful Investigations' which showed some very slight increase in Administrative' decisions towards the end of the process. This might possibly be accounted for as a reasonable consequence of the investigative process during an initial investigation, where administrative 'events' in the form of routine issues, checks and balances naturally fall as such investigations move towards closure.

Decision 'Cause' over Time : Visualisation of data for the three sub-categories of decision 'causation' showed two broadly different patterns for different case types. Specifically, 'unsuccessful investigations' showed a high frequency of 'proactive' decisions early in the investigation, with no 'proactive' decisions whatsoever in the middle or latter stages; these same investigations showed higher levels or 'reactive' decisions throughout the full investigation. By contrast, both 'potentially successful' investigations' and 'wrongful' investigations showed high proportions of both 'proactive' and 'reactive' decisions across the whole period of the investigative process. This may infer a correlation between 'proactive' decisions across the full investigative process and 'success' of the investigation.

Decision 'Intent' and 'Connectivity' over Time : Examination of the patterns in all three sub-categories of decision 'Intent' and 'Connectivity' showed broadly the same pattern regardless of case type, namely that all sub-categories were distributed evenly over the whole period of the investigative process, with no other discernible pattern.

Decision 'Creativity' over Time : Visualisation of data for the three sub-categories of decision 'Creativity' showed two broadly different patterns for different case types (Appendix C). Specifically, 'unsuccessful investigations' showed a high frequency of 'creative' decisions early in the investigation, but with relatively far fewer 'creative' decisions in the middle or latter stages. By contrast, both 'potentially successful' investigations' and 'wrongful' investigations showed high proportions of both 'creative' decisions across the whole period of the 
investigative process. This may infer a correlation between 'creative' decisions across the full investigative process and 'success' of the investigation.

Decision 'Impact' over Time : Examination of the patterns in all three sub-categories of decision 'Impact' showed broadly the same pattern regardless of case type, namely that 'impactive' decisions were distributed evenly over the whole period of the investigative process, with no other discernible pattern. However, as regards the nature of the 'impact', where it was identified, some different patterns were identified. Firstly, in the case of decisions with 'positive' Impact, (Chart C), broadly speaking such decisions were far more evenly spread across the investigative timeline in both 'potentially successful' investigations' and 'wrongful' investigations than in 'unsuccessful' investigations, where they tended to appear only in the earlier and latter stages of the investigation. Secondly, in the case of decisions with 'negative' Impact, in both 'unsuccessful' and 'potentially successful' investigations, negative decisions broadly tended to cluster towards the mid-point and latter stages of the investigation timeline, whilst in the case of 'wrongful' investigations, decisions with a negative impact were more evenly spread across the whole investigative timeline. Given that both 'potentially successful' and 'wrongful' case types by their nature each featured unsuccessful outcomes in the earlier parts of their investigation, followed by 'successful outcomes in the later parts, that this trend broadly correlated with investigative 'success' is perhaps hardly surprising.

Thus specifically regarding changes in decision categories over time and their correlation with investigative 'success', whilst not all categories demonstrated any notable time-relevant correlation, some did, namely:

- The ongoing presence over time of 'proactive' decisions appeared to correlate positively with 'success'. 
- The ongoing presence over time of 'creative' decisions appeared to correlate positively with 'success'.

This implies that the generation throughout the investigation of fresh thoughts or ideas, including creative ideas that think 'outside the box', are both factors in overall investigative 'success'. Further, as regards 'impact', the changes of both positive and negative 'impact 'over time appears to follow the presence (or absence) of 'proactive' and/or 'creating' decisions. This may suggest then that 'impact', positive or negative, is a consequence of the presence (or not) of 'proactive' and/or 'creative' decisions, rather than a driver that influences investigative success in its own right.

\section{Identification of 'Tipping Points'}

In their studies of sequential decision-making by SIO's, Fahsing \& Ask (2013) identified 'tipping points' - points in time when the mind-set of the SIO 'switched' from 'exploratory' (seeking to identify a suspect or suspects) to 'verification' (seeking to verify a suspect's involvement in the crime). Whilst a potentially significant finding, it is important to acknowledge that the methodology of this research was limited to seeking SIO responses to wholly artificial cases, and moreover sought only to identify 'tipping points' solely based purely on the 'Suspect Identification/Suspect Verification' criterion, thus any findings were arguably also similarly limited by these parameters.

The decision processes in the case studies captured in this research were therefore also examined for similar 'tipping points', and in every case a tipping point was identified, either side of which investigative decisions and outcomes clearly differed once a suspect or suspects were identified. This would tend to infer that the findings of the earlier study might also be found in real homicide investigations. 
Contemporaneous decision categorisation also enabled comparisons between 'tipping points' and changes in decision categories, which presented a number of interesting observations beyond those of the study conducted by Fahsing and Aske. First, changes over time identified in 'positive' and 'creative' decisions correlated closely with 'tipping points'; specifically the presence of high proportions of such decisions after tipping points broadly correlated with investigative 'success', whilst their absence after tipping points broadly correlated with investigative 'failure'. Secondly, in addition to what might be termed 'suspect-focussed' tipping points identified by the earlier study, this study also identified other, different 'tipping point' focuses, including fresh evidence and fresh organisational perspectives/triggers. Further, these 'other' tipping point focuses also exhibited broad correlations with 'creative' and 'proactive' decision categories.

This suggests that a range of factors both within and outside investigations may act as 'tipping points', and that regardless of cause, each 'tipping point' correlates with changes in the relative proportion of 'proactive' and 'creative' decisions. It also supports the earlier inference that 'proactive' and/or 'creative' decisions can stimulate investigative 'success'. Thus the mental approach of the SIO could itself act as a 'trigger' 'internal to the investigation to stimulate investigative 'success'.

\section{Identification of 'Transition Points'}

By their very nature, the case studies incorporated investigative timelines that spanned more than just an unsuccessful initial reinvestigation, covering reviews and re-investigation, all part of the 'investigative continuum' of an individual case. That being so, the 'transition point', namely the point in each investigation where the process is subject to change, be that initiation or closure of an investigation, or initiation/closure of a review, may be of interest. With that in mind, every 'transition point' in each case was highlighted in terms of its position in the overall sequence of events for that case (Chart D). This enabled relative comparisons of the position in time of a 'transition point', firstly to categories of individual decisions and any changes in 
them, and secondly in relation to 'tipping points'. Multiple 'transition points' were identified, and their impact on decision categories and investigative 'success' was found to largely mirror that of 'tipping points'. Further, whilst there appeared to be little correlation between 'transition' and 'tipping points' overall, where the two did coincide in an investigative timeline, the impact in terms of subsequent positive outcomes or investigative 'success' was improved. This may infer that organisational or process intervention in an investigative continuum may well also act as an external 'trigger' to stimulate investigative 'success'.

\section{Conclusions}

This research sought to categorise investigative decisions according to how they operated and to compare the points in time they were made against other case data, in an attempt to explore how different decision categories might have impacted upon the course of the investigation and to try and identify if different decision 'patterns' preceded changes in the course of an investigation that led to successful outcomes, or not, the inference being that any such changes were, in part at least, linked to, or caused by the decision patterns.

In general terms, this research suggests that greater investigative 'success' may be linked to:

- Greater total numbers of decisions

- Greater relative proportions of 'investigative' and 'descriptive' decisions

- Higher proportions of 'proactive' over 'reactive' decisions

- Higher proportions of 'expansive/explorative' over 'regressive' decisions

- Higher proportions of 'impactive' decisions in general, and specifically of 'positively impactive' decisions

- The on-going presence of 'proactive' and 'creative' decisions.

Thus throughout an investigative timeline, -facilitated in this research by the novel conceptual framework of the 'investigative continuum', the generation of fresh thoughts or ideas, 
particularly creative ideas that think 'outside the box', are apparently factors in improving overall investigative 'success'. Further, the changes of both positive and negative 'impact 'over time appears to correlate positively with 'proactive' and/or 'creative' decisions. This may suggest that 'impact', positive or negative, is a consequence of the presence, or not, of 'proactive' and/or 'creative' decisions, rather than a driver that influences investigative success in its own right.

This research also corroborates the presence and impact of 'tipping points' identified in other earlier research, within real investigations, and extends understanding of how these can relate to, and impact, both positively and negatively, on decisions and on outcomes. In every case a tipping point was identified either side of which in time the investigative decisions and outcomes clearly differed once a suspect or suspects were identified. Firstly, the changes over time identified in 'positive' and 'creative' decisions correlated closely with tipping points; specifically the presence of high proportions of such decisions after tipping points broadly correlated with investigative 'success', whilst their absence after tipping points broadly correlated with investigative 'failure'. Secondly, in addition to what might be termed the 'suspect-focussed' tipping points identified in other studies, this research also identified other, different 'tipping point' focuses, including fresh evidence and fresh organisational perspectives/triggers; moreover, these 'other' tipping point focuses also exhibited broad correlations with 'creative' and 'proactive' decision categories.

This research also identified and conceptualised the new notion of 'transition points' - points in the investigative lifetime or continuum of an investigation where the process is subject to change - be that initiation or closure of an investigation, or initiation/closure of a review. Across the 'investigative continuum' of every case multiple 'transition points' were identified. Whilst there appeared to be little direct correlation between transition points and changes in decision categories over time, or even between 'transition' and 'tipping points' overall, the occurrence of transition points was generally followed by positive outcomes. Further, where 
the two did coincide in an investigative timeline, the impact in terms of subsequent positive outcomes or investigative 'success' was also improved.

Thus, in the 'investigative continuum' of a case transition points, both in isolation and in combination with tipping points', through their consequential impact on decision-making generally precede improvements in positive outcomes and investigative 'success' than those correlating with 'tipping points' alone.

The limited number of cases, coming from a single Force, must limit the generality of these research findings to be, at best, indicative rather than in any way conclusive. Nevertheless, the validity in the methodology of case selection, decision 'categorisation', the novel perspective of an 'Investigative Continuum', and the focus on real cases, arguably offer methodologies worthy of consideration to researchers contemplating similar research, perhaps with wider and more robust data samples. 
TABLES \& CHARTS

Table 1 : Decision Data- Categorisation Matrix

\begin{tabular}{|l|l|l|}
\hline CATEGORY & SUB-CATEGORY & Description \\
\hline Objective & $\begin{array}{l}\text { Descriptive; } \\
\text { Administrative; } \\
\text { Investigative }\end{array}$ & The functionality of the decision - its purpose \\
\hline Intent & Proactive: Reactive & $\begin{array}{l}\text { The cause of the decision -an active product of creative } \\
\text { thought or a passive reaction to new information }\end{array}$ \\
\hline Connectivity & $\begin{array}{l}\text { Legressive } \\
\text { Linked : Consequential }\end{array}$ & $\begin{array}{l}\text { The intent of the decision; intended to open up or to } \\
\text { independent, or a decision leading directly to others } \\
\text { rather than their arising independently }\end{array}$ \\
\hline reduce, limit, or finalise an element of the investigation \\
\hline Impact & Identifiable & $\begin{array}{l}\text { The relationship between information as a stimulus for } \\
\text { a decision and the decision itself. Was it the clear result } \\
\text { of specific information, or the apparent result of 'creative' } \\
\text { thought? }\end{array}$ \\
\hline Yes - Positive ; Negative & $\begin{array}{l}\text { The impact and outcome of a decision on the } \\
\text { investigation ; was there an apparent impact or not, and } \\
\text { if so, was it 'positive' (contributory towards a successful } \\
\text { conclusion), or 'negative' (detrimental to a successful } \\
\text { conclusion) }\end{array}$ \\
\hline
\end{tabular}

\section{Chart A : Quantitative Summaries}

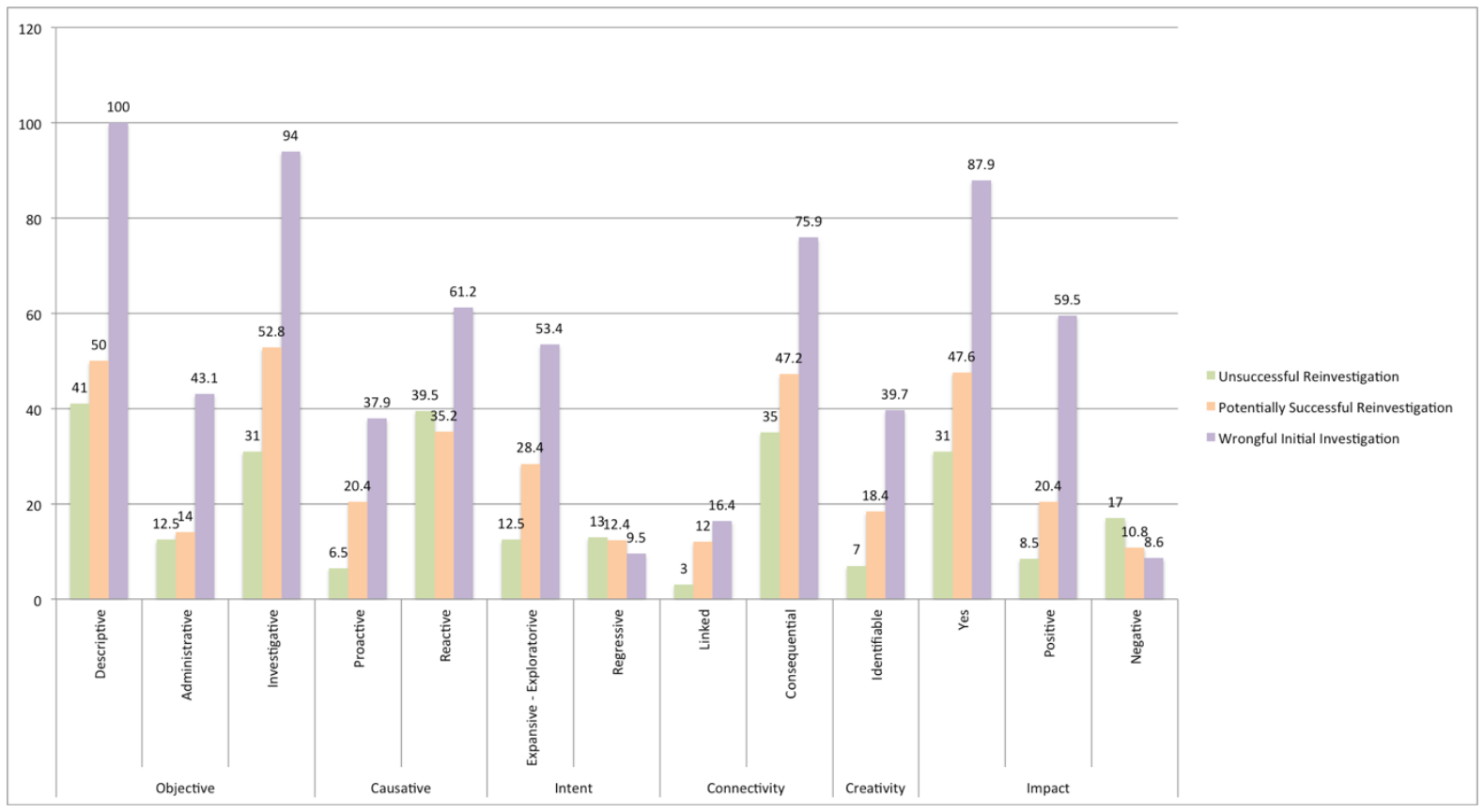


Chart B : Comparative Range over Time of 'Creative' Decisions by Case Type

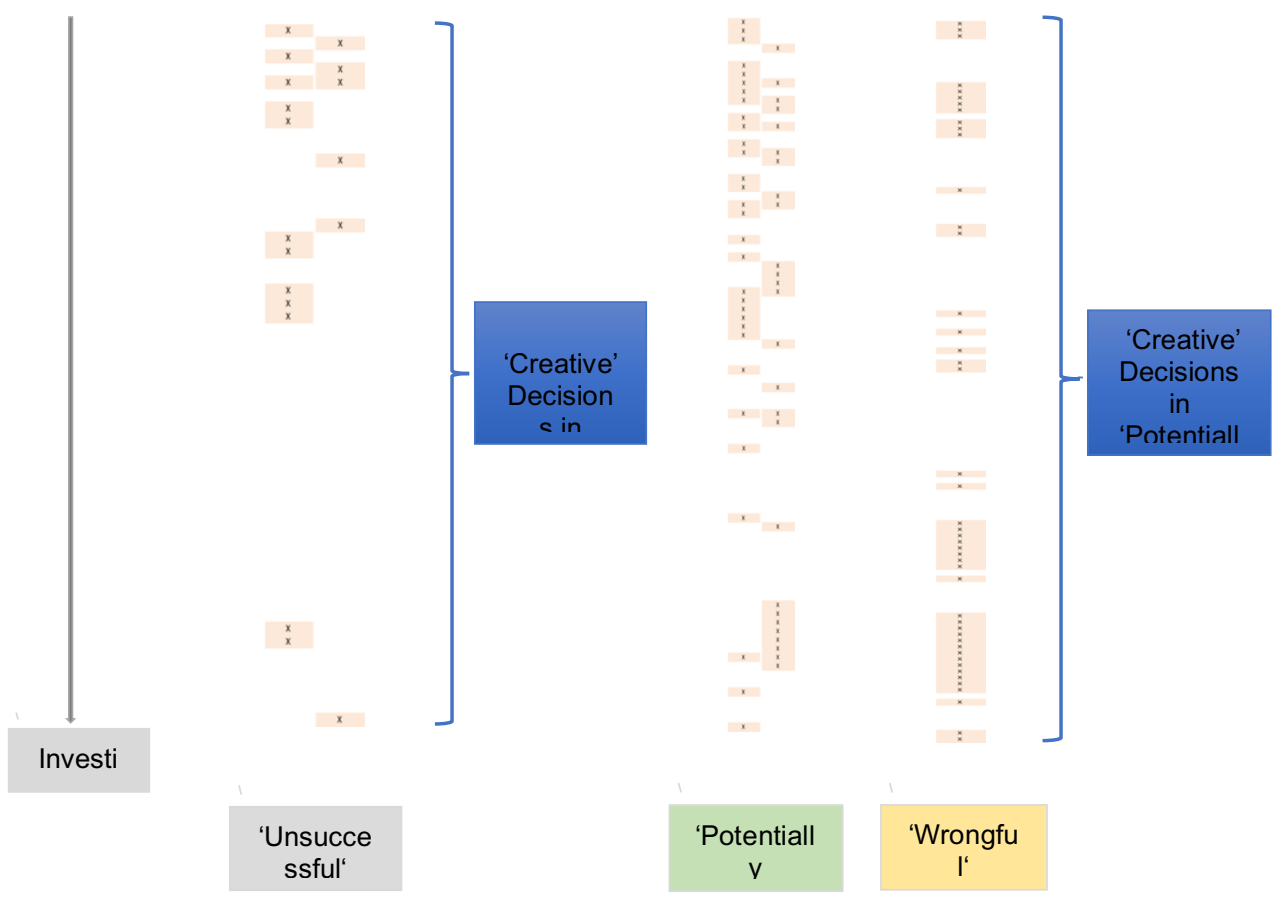

Chart C : Comparative Range over Time of 'Positive Impact' of Decisions by Case Type

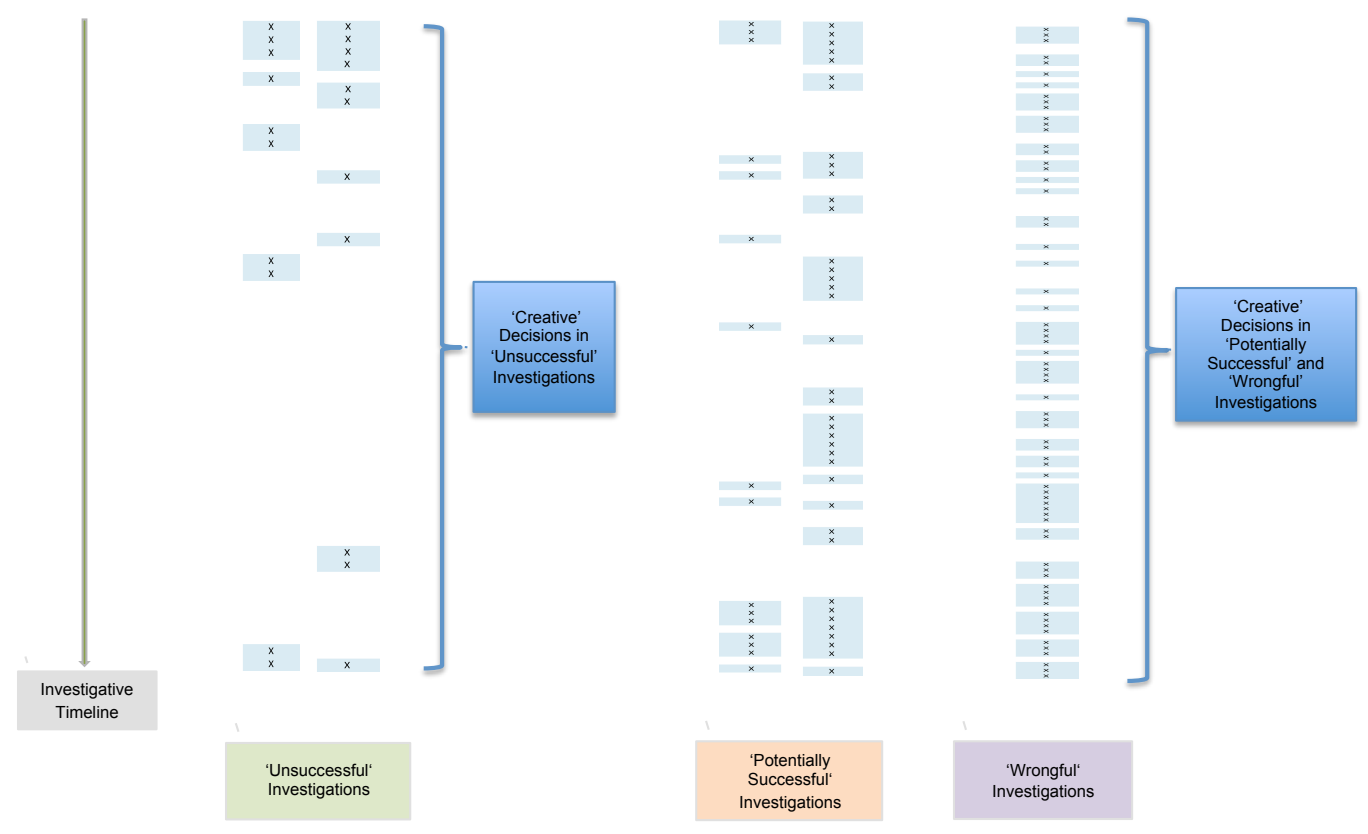




\section{REFERENCES}

ACPO. (2010). Major Crime Reviews - ACPO Guidance (Draft Version 1). London, ACPO.

ACPO. (2005). Practice Advice on Core Investigative Doctrine. London, ACPO.

ACPO. (2006). Murder Investigation Manual. National Centre for Policing Excellence, (NCPE) Wyboston, UK.

ACPO. (2012). The National Decision Model. National Centre for Policing Excellence. Wyboston, UK.

Bryman, A. (2016). Social Research Methods, Oxford, UK.

Byford, L. (1981). The Yorkshire Ripper Case : Review of the Police Investigation of the Case. Report presented to the Secretary of State for the Home Department, December 1981.

Copi, I.M., \& Cohen, C (1998). Introduction to Logic . Prentice Hall

Corbin, J, \& Strauss, A. (2015). Basics of Qualitative Research (Fourth Edition), pp. 4-6. Sage, UK.

Dror, I.E., Busemeyer, J.R., \& Basola, B. (1999). Decision making under time pressure: An independent test of sequential sampling models. Memory \& Cognition, 27(4), pp. 713-725, Psychonomic Society Inc. 
Fahsing, I. \& Ask, K. (2013). Decision Making and Decisional Tipping Points in Homicide Investigations: An Interview Study of British and Norwegian Detectives. Journal of Investigative Psychology and Offender Profiling ${ }_{2}$ 10. Pp. 155-175. Wiley, UK.

Fahsing, I. \& Ask, K. (2015). The making of an expert detective : the role of experience in English and Norwegian police officers' investigative decision-making. Psychology, Crime \& Law $22: 3$, Pp.203-233. UK.

Fisher, A. (2001). Critical Thinking : An Introduction. Cambridge University Press.

Glaser, B.G., \& Strauss, A.L. (1967). The Discovery of Grounded Theory; Strategies for Qualitative Research. Aldine, USA.

Heller, R. (1998). Making Decisions. Dorling Kindersley.

Heuer, R.J. (1999). Psychology of Intelligence Analysis. Center for the Study of Intelligence, Central intelligence Agency, USA.

Innes, M. (2002). The 'Process Structures' of Police Homicide Investigations. The British Journal of Criminology ${ }_{2}$ Vol. 42, Issue 4, P. 669-688.

Innes, M.R., \& Brookman, F. (2013). Helping Police with their enquiries : International perspectives on homicide investigation. Policing and Society : an International Journal of Research and Policy, $23: 3$, pp. 285-291.

Kahneman, D., \& Tversky, A. (2000). Choices, Values and Frames. Cambridge University Press. 
Macpherson, W. (1999). The Stephen Lawrence Enquiry, February 1999. Stationery Office.

Nicol, C., Innes, M., Gee, D., \& Feist, A. (2004). Reviewing murder investigations : an analysis of progress reviews from six police forces. Online Report 25/04, UK Home Office.

Popper, K. (1972). The Logic of Scientific Discovery. Routledge.

Roach, J. (2017). The Retrospective Detective. Cognitive Bias and the Cold Case Homicide Investigator. Papers from the British Criminology Conference, Vol.17.

Roach, J. (2012). Long Interval Detections and Under the Radar Offenders. Journal of Homicide and Major Incident Investigation 8 (1). pp. 20-38.

Roach, J., and Pease, K. (2009). Necropsies and the cold case. D.K. Rossmo (Ed.) (2009). Criminal Investigative Failures, Boca Raton: CRC Press.

Rossmo, D.K. (2006). Criminal Investigative Failures : Avoiding the Pitfalls (parts $1 \& 2$ ). In FBI Law Enforcement Bulletin 75 (9), September, pp. 1-10, and FBI Law Enforcement Bulletin 75 (10), October, pp. 12-19.

Rossmo, D. K. (2008). Criminal Investigative Failures. USA. CRC Press.

Roycroft, M. (2009). Solving factors and decision making in 'hard to solve' murder enquiries. PhD Thesis (unpublished). University of Surrey, UK.

Smith, J. (2005). The Shipman Enquiry. Report to the Secretary of State for Health, MH Government UK. (http://webarchive.nationalarchives.gov.uk/20090808155114/http://www.theshipman-inquiry.org.uk ; accessed 24/10/17) 
Stelfox, P. (2006). The Factors that determine Outcomes in the Police Investigation of Homicide. PhD Thesis (unpublished). Open University, UK

Stelfox, P., \& Pease, K. (2005). Cognition and detection : Reluctant bedfellows ? M. Smith \& N. Tilley (Eds.) (2005) Crime science: New approaches to preventing and detecting crime. Willan Publishing.

Weisburd, D, Telep, C, Hinkle, J \& Eck, J (2008). The effects of Problem-Oriented Policing on Crime and Disorder. US Department of Justice, Office of Justice Programs, National Institute of Justice, USA. 